\title{
Supporting Information \\ Oxidation of Gallium-Based Liquid Metal Alloys by Water
}

Megan A. Creighton, ${ }^{1,3}$ Michelle C. Yuen,,3 Michael A. Susner, ${ }^{2,3}$ Zachary Farrell, ${ }^{2,3}$ Benji

Maruyama, ${ }^{3}$ Christopher E. Tabor*3

*Corresponding author, christopher.tabor.1@us.af.mil

1. National Research Council, Washington, DC 20001, United States

2. UES, Inc., Dayton, OH 45431, United States

3. Materials and Manufacturing Directorate, Air Force Research Laboratory, Wright-Patterson Air Force Base, Dayton, $\mathrm{OH} 45433$, United States

Number of pages: 8

Number of figures: 8

Number of schemes: 0

Number of tables: 3

\section{Table of Contents:}

1. XRD analysis of the crystallites

2. LMP Preparation in oxygen-free conditions using Novec ${ }^{\mathrm{TM}} 7100$ :

3. LMP surface chemistry analysis by XPS: 


\section{XRD analysis of the crystallites:}

Using powder X-ray diffraction (XRD), we identified the grey-white particles as $\mathrm{GaOOH}$. The observed results are in accord with other reports found in the literature. ${ }^{1-3}$ The crystallites studied were formed using both eGaIn and another low-melting alloy containing tin $(68.5 \% \mathrm{Ga}, 21.5 \%$ In, $10 \% \mathrm{Sn}$ ) as the liquid metal source. Interestingly, only the orthorhombic phase (a grey-white powder) was discernible in the pattern for the former case. Figure S1 shows the diffraction data together with the Rietveld model and the $\chi^{2}$ for the fit (3.19) for the crystallites as a byproduct of eGaIn LMP formation. The collected XRD data (red circles), Rietveld fit (black line), difference plot (blue line), and peak reflection locations (green vertical lines) are all shown. The inset shows the crystal structure of $\mathrm{GaO}(\mathrm{OH})$ with the green spheres representing the gallium, the blue spheres representing the oxygen, and the red spheres representing the hydrogen. Figure S2 shows the collected XRD data for when the $\mathrm{Ga} / \mathrm{In} / \mathrm{Sn}$ alloy is used in lieu of eGaIn.

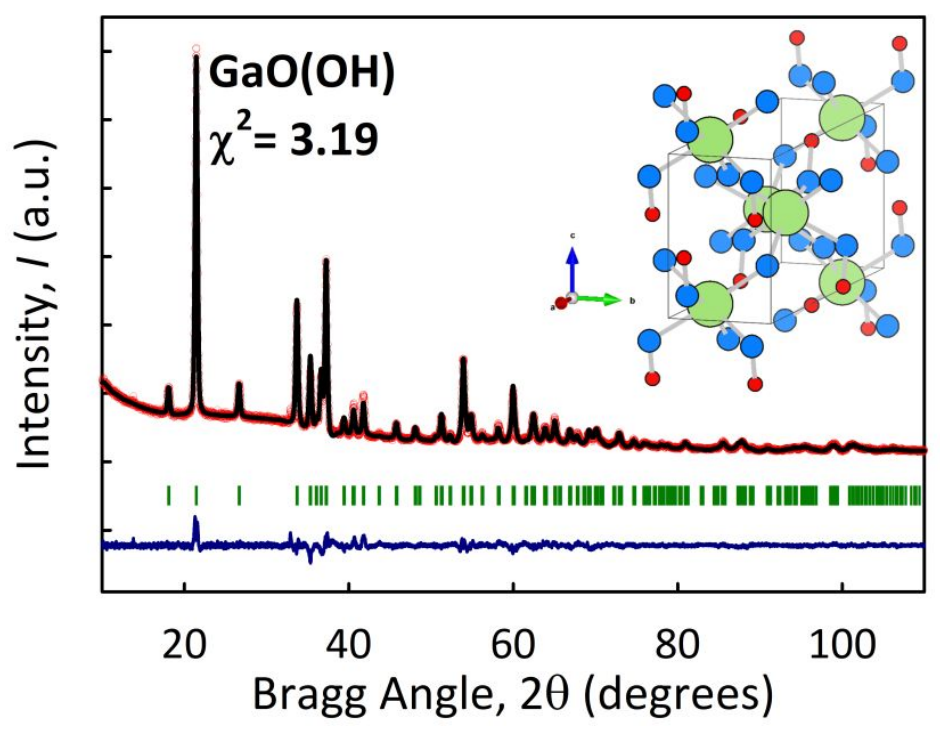

Figure S1. Rietveld refinement of crystallites formed from the eGaIn liquid metal source showing XRD data (red circles), Rietveld fit (black line), difference plot (blue line), and peak reflection locations (green vertical lines) for the labeled phases.

In Table 1 we show the refinement values for the atomic coordinates and the unit cell for the sample derived from eGaIn. The second sample was derived from the aforementioned tin-containing alloy. It contained $\mathrm{Sn}, \mathrm{Ga}$, and $\mathrm{In}$ as the main metallic elements and again yielded $\mathrm{GaO}(\mathrm{OH})$ as the main phase present. However, two impurity phases were also evident: $\operatorname{In}_{0.2} \mathrm{Sn}_{0.8}$ and $\mathrm{In}_{0.75} \mathrm{Sn}_{0.25}$. Given the binary phase diagram of the In-Sn system (e.g. Bartram et $\mathrm{al}^{4}$ ) this is unsurprising as these two phases coexist for $0.3<\mathrm{Sn}<0.7$ in the temperatures of interest. We show the Rietveld refinement in this multiphase sample in Figure S1 together with the Rietveld refinements in Tables S1-S3. 
Table S1. $\mathrm{GaO}(\mathrm{OH})$ Rietveld refinement parameters for eGaIn sample.

\begin{tabular}{lllll}
\hline Atom & \multicolumn{3}{c}{ Atomic Coordinates } & Occ. \\
\cline { 2 - 5 } & $\mathrm{x}$ & $\mathrm{y}$ & $\mathrm{z}$ & \\
$\mathbf{G a 1}$ & $0.14243(13)$ & $3 / 4$ & $0.95371(25)$ & 1 \\
$\mathbf{H 1}$ & 0.08795 & $1 / 4$ & 0.40537 & 1 \\
$\mathbf{O 1}$ & $0.05921(51)$ & $1 / 4$ & $0.16603(98)$ & 1 \\
$\mathbf{O 2}$ & $0.19473(52)$ & $1 / 4$ & $0.7221(10)$ & 1 \\
\hline
\end{tabular}

\section{Fitting Parameters}

$\begin{array}{llll}\chi^{2} & \mathrm{R}_{\mathrm{p}} & \mathrm{R}_{\mathrm{wp}} & \mathrm{R}_{\text {exp }}\end{array}$

$\begin{array}{llll}3.19 & 4.56 & 5.96 & 3.38\end{array}$

Space Group: Pnma (No. 47)

$\begin{array}{llll}\text { a: } 9.81787(21) \AA & \text { b: } 2.97134(6) \AA & \text { c: } 4.57409(14) \AA \quad Z=4\end{array}$

$\alpha: \mathbf{9 0}^{\circ} \quad \beta: 90^{\circ} \quad \gamma: 90^{\circ} \quad V=133.408(8) \AA^{3}$

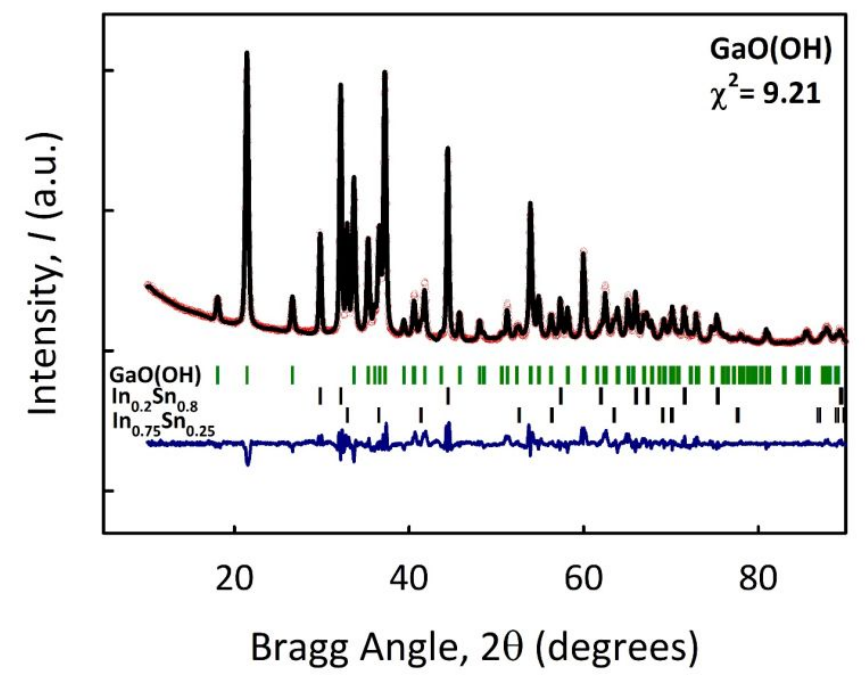

Figure S2. Rietveld refinement of the $68.5 \% \mathrm{Ga}, 21.5 \% \mathrm{In}, 10 \% \mathrm{Sn}$ alloy showing XRD data (red circles), Rietveld fit (black line), difference plot (blue line), and peak reflection locations (green vertical lines) for the labeled phases. 
Table S2. $\operatorname{In}_{0.2} \mathrm{Ga}_{0.8}$ Rietveld refinement parameters for Sample 2 .

\begin{tabular}{lcccc}
\hline Atom & \multicolumn{3}{c}{ Atomic Coordinates } & Occ. \\
\cline { 2 - 5 } & $\mathrm{x}$ & $\mathrm{y}$ & $\mathrm{z}$ & \\
Sn1 & 0 & 0 & 0 & 0.8 \\
In1 & 0 & 0 & 0 & 0.2
\end{tabular}

\section{Fitting Parameters (phase)}

\begin{tabular}{lll}
$\chi^{2}$ & $\mathrm{R}_{\mathrm{f}}$ & $F_{\mathrm{In} 0.2 \mathrm{Ga} 0.8}$ \\
$\mathbf{3 . 9 9}$ & 2.70 & $0.1228(12)$ \\
\hline
\end{tabular}

\section{Fitting Parameters (total)}

$\begin{array}{llll}\chi^{2} & \mathrm{R}_{\mathrm{p}} & \mathrm{R}_{\mathrm{wp}} & \mathrm{R}_{\text {exp }} \\ \mathbf{9 . 2 0} & 6.93 & 9.04 & 2.99\end{array}$

\section{Space Group: P6/mmm (No. 191)}

\begin{tabular}{lll} 
a: 3.21366(9) $\AA$ & b: $3.21366(9) \AA$ & c: $2.99597(12) \AA$ \\
$\alpha: \mathbf{9 0}^{\circ}$ & $\beta: 90^{\circ}$ & $\gamma: 120^{\circ}$ \\
\hline
\end{tabular}

Table S3. $\operatorname{In}_{0.75} \mathrm{Ga}_{0.25}$ Rietveld refinement parameters for Sample 2.

\begin{tabular}{lcccc}
\hline Atom & \multicolumn{3}{c}{ Atomic Coordinates } & Occ. \\
\cline { 2 - 5 } & $\mathrm{x}$ & $\mathrm{y}$ & $\mathrm{z}$ & \\
Sn1 & 0 & 0 & 0 & 0.25 \\
In1 & 0 & 0 & 0 & 0.75
\end{tabular}

\section{Fitting Parameters (phase)}

$\begin{array}{lll}\boldsymbol{\chi}^{2} & \mathrm{R}_{\mathrm{f}} & F_{\mathrm{In} 0.75 \mathrm{Ga} 0.25} \\ \mathbf{3 . 1 4} & 2.68 & 0.0405(9)\end{array}$




\section{Fitting Parameters (total)}

$\begin{array}{llll}\chi^{2} & \mathrm{R}_{\mathrm{p}} & \mathrm{R}_{\mathrm{wp}} & \mathrm{R}_{\exp } \\ \mathbf{9 . 2 0} & 6.93 & 9.04 & 2.99\end{array}$

\section{Space Group: $P 6 / \mathrm{mmm}$ (No. 191)}

$$
\text { a: 3.47931(30) } \AA \quad \text { b: } 3.47931(30) \AA \quad \text { c: } 4.36746(62) \AA
$$
$\alpha: 90^{\circ}$
$\beta: 90^{\circ}$
$\gamma: 120^{\circ}$

\section{LMP Preparation in oxygen-free conditions using Novec ${ }^{\mathrm{TM}} 7100$ :}

Similar to the case with anoxic, anhydrous ethanol, the vast majority of the metal remains in bulk phase (Figure S3A), and the particles that do form have a very large average diameter (10.78 um $\pm 2.08 \mathrm{um}$ in degassed conditions, compared to $0.74 \pm 2.35 \mathrm{um}$ in ambient) and many cases of partial coalescence can be observed. (Figure S3B).

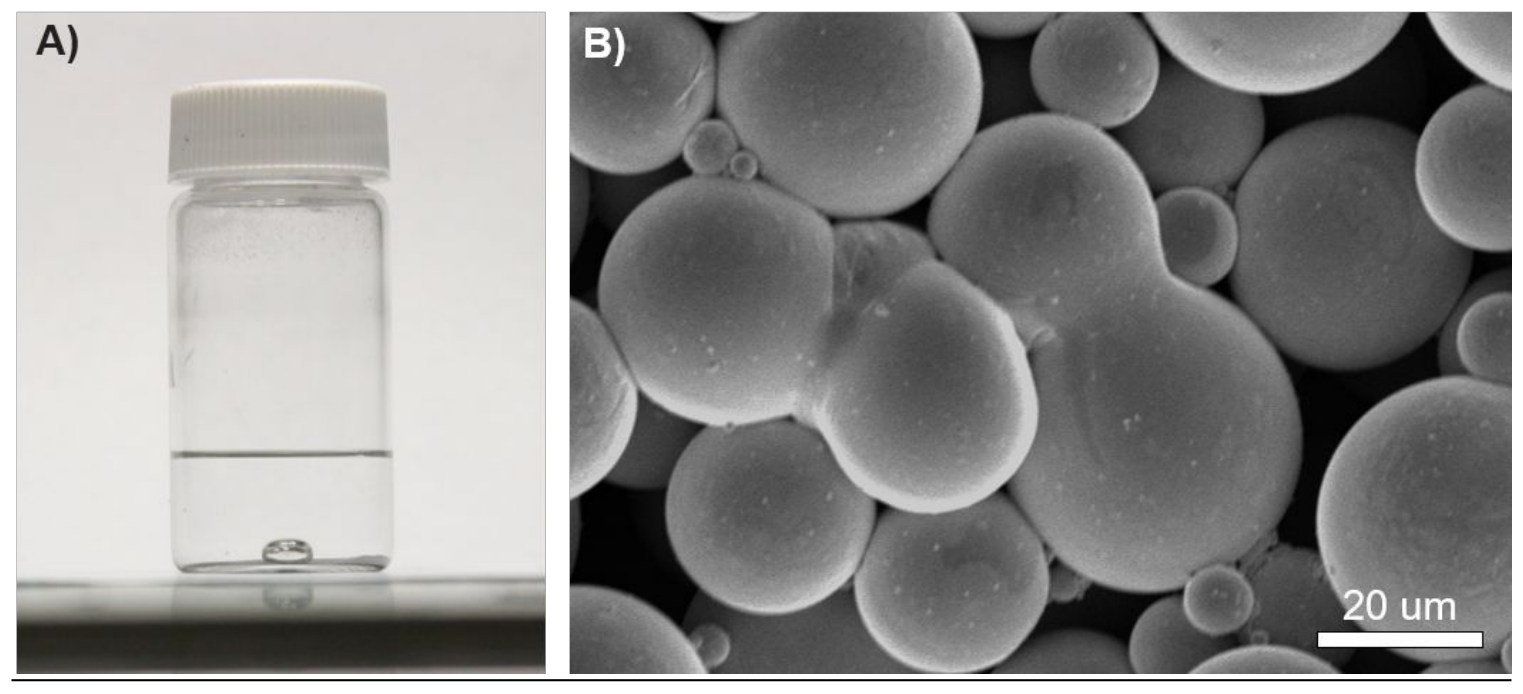

Figure S3. Formation of LMPs in anhydrous, anoxic conditions. A) When Novec ${ }^{\mathrm{TM}} 7100$, a known inert solvent, is used, a similar majority of the liquid metal volume remains in bulk form. B) Partial coalescence of neighboring particles is observed, similar to the ethanol case.

\section{LMP surface chemistry analysis by XPS:}

Spectra were analyzed using the CasaXPS software program, version 2.3.22PR1.0 (Casa Software Ltd.). A Tougaard background was subtracted from each region before curve fitting; components were fitted with Gaussian-Lorentzian peaks of CasaXPS Lineshapes of GL(30). The spacing of spin-orbit coupling doublets were constrained to $0.85 \mathrm{eV}$ for In and $0.45 \mathrm{eV}$ for $\mathrm{Ga} .{ }^{5}$ The full width half maximum (FWHM) of the doublet peaks were constrained to match.

The binding energy region shown in Figure S4 contains contributions from multiple elements of interest ( $\mathrm{Ga3} d, \mathrm{In} 4 \mathrm{~d}$, and $\mathrm{O} 2 \mathrm{~s}$ ). All indium present can be assumed to be in the 
metallic state, as gallium creates the passivating skin layer. ${ }^{6,7}$ The oxygen peak (red) is constrained in size by converting the size of the measured O1s peak using the respective relative sensitivity factors. The relative size of the oxidized species peak (blue) to the $\mathrm{Ga}^{0}$ metal peaks (green) shows a clear trend with decreasing trend with decreasing water concentration (Figure S4A-C). The spacings between all peaks representing the various species were fixed relative to $\mathrm{In}^{0}$ except for the oxidized gallium species, which were allowed to float. Note that the doublet peak for the oxidized species is contained fully within the primary peak, and could be fit equally well by a single peak. There are several degrees of freedom regarding this region, and not enough information is available to discern if there are multiple oxidized species present with overlapping spectra.

These peaks representing the oxidized species shift $0.1 \mathrm{eV}$ higher when the solvent is changed from pure ethanol to $75 \%$ ethanol and $25 \%$ water, and an additional $0.1 \mathrm{eV}$ higher as the solvent ratio is further changed to $50 \%$ ethanol and $50 \%$ water. This trend is consistent with the formation of $\mathrm{GaO}(\mathrm{OH})$ species and/or hydrated oxides. ${ }^{8}$ No more shifting is observed as the solvent ratio continues to increase towards pure ethanol. The Novec ${ }^{\mathrm{TM}} 7100$ solvent (panel D) spectra has a very large peak for the oxidized species, but the spacing between the peaks is consistent with the ethanol sample, further supporting the hypothesis that different oxidized species are formed in the presence of water.

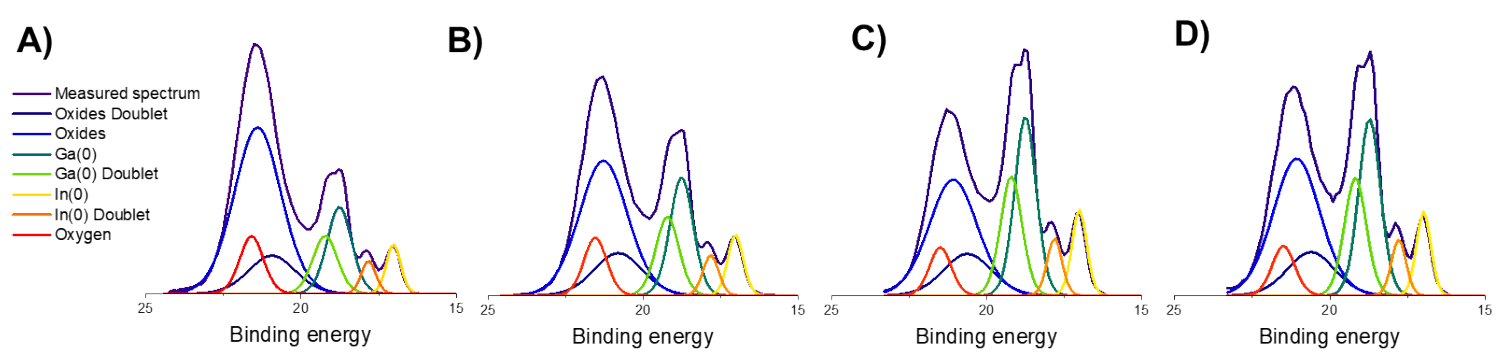

Figure S4. XPS spectra in the Ga3d region of eGaIn LMPs made in the presence of varying solvents. A) Pure water, B) 50\% water and 50\% ethanol (v/v), C) pure ethanol, D) Novec ${ }^{\mathrm{TM}} 7100$.

The increase in oxide content can also be verified by the indium signal. Indium has a lower redox potential, and becomes protected from oxidation by the outer layer of gallium oxides. ${ }^{6,7}$ Therefore, all indium signal comes from the core. The measured spectrum is from a limited depth of penetration from the surface that becomes weaker as the oxide skin becomes thicker and dominates the outgoing signal. The trend is shown in Figure S5. 


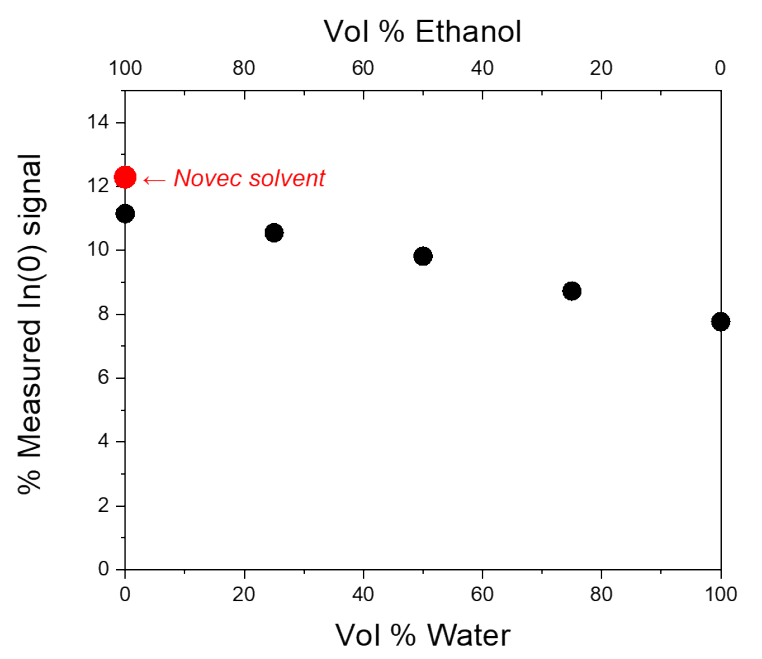

Figure S5. Decreasing indium signal with increasing gallium oxide signal

The LMPs made in the Novec ${ }^{\mathrm{TM}} 7100$ have a similar surface chemistry profile to those made in ethanol (Figure S6). In this figure, the relative amount of gallium oxides to gallium metal is determined by the Ga3s region, and the relative amount high binding energy (HBE) oxides to low binding energy (LBE) oxides is determined from the O1s peak. (See Figure 4, main text.) The oxide content for the Novec ${ }^{\mathrm{TM}} 7100$ samples is slightly higher overall, with a slightly larger portion of LBE oxide. This can also be seen by comparing the indium signals of the two samples in Figure S4. The solubility of gas molecules is significantly higher in fluorosolvents than in comparative hydrocarbon solvents, ${ }^{9}$ and so this effect is likely driven by an advantage in mass transfer. The LMPs made in water have the smallest ratio of gallium metal, and a smaller proportion of the oxide formed is attributed to the LBE oxide.

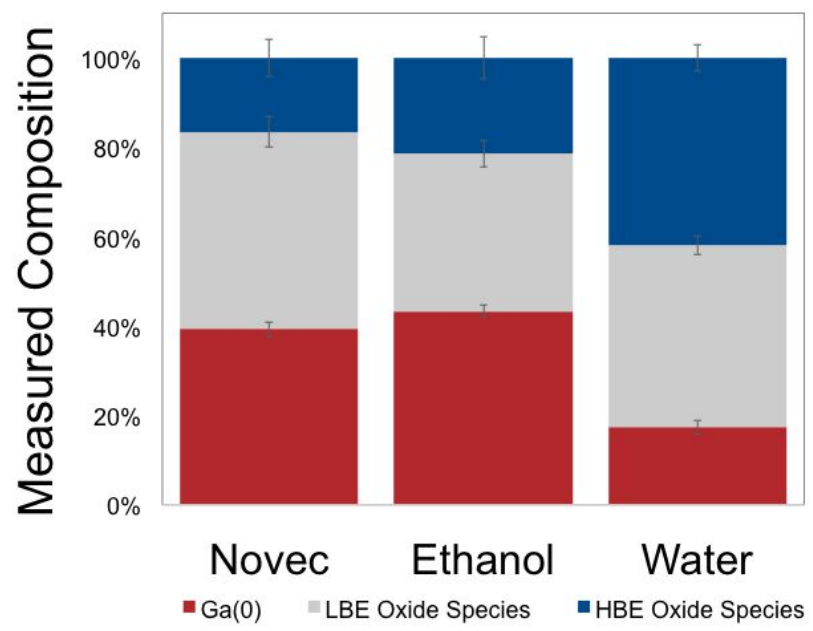

Figure S6. Comparison of eGaIn LMP surfaces when pure water, ethanol, or Novec ${ }^{\mathrm{TM}} 7100$ is used as the processing solvent, as determined by XPS analysis.

Samples that have been exposed to ambient conditions are known to develop a thin layer of carbonaceous debris known as adventitious carbon, generally regarded to be only a few nanometers thick. Lin et al have previously reported that using ethanol as a processing solvent leads to the formation of a carbon shell surrounding the LMPs. ${ }^{10}$ While Lin and co-authors 
acknowledge this shell could be composed of adventitious carbon, they further show that it can be observed by TEM only for samples processed in ethanol. In their work, samples processed in other hydrocarbons such as dodecane do not exhibit the same phenomenon.

All samples studied in the work presented here show evidence of an adventitious carbon layer as measured by XPS (Figure S7). However, in our study the relative percentage of carbon in the samples studied did not show any trends caused by the composition of the processing solvents used. Despite this consistency in the carbon level among the samples, the oxygen content of the samples show a clear increase in relation with the fraction of water.

\section{$\%$ Ethanol $(\mathrm{v} / \mathrm{v})$}

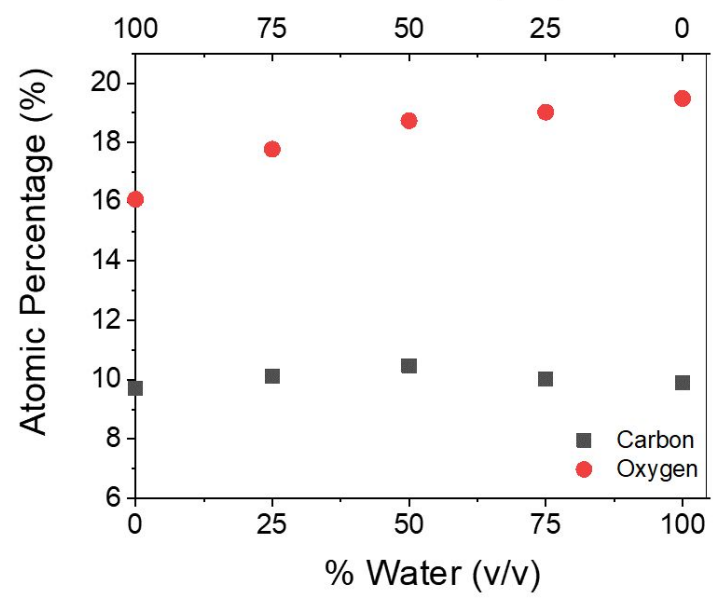

Figure S7. XPS measurements show that the oxygen content in all samples studied is influenced by the relative ratios of water and ethanol in the processing solvent, but there are observable patterns in the carbon content.

Intrigued by the observations of Lin et al, we further examined the C1s spectra of our samples to see if there were any changes in the nature of the adventitious carbon layer despite its consistency in thickness. The spectra for samples prepared in water and ethanol are shown in Figure S8. The features between the two cases are very consistent. The most prominent peak is the characteristic C-C peak $(284.8 \mathrm{eV})$, and the peaks for $\mathrm{C}-\mathrm{O}(\sim 286.6 \mathrm{eV})$ and $\mathrm{O}-\mathrm{C}=\mathrm{O}(\sim 289.2$ $\mathrm{eV}$ ) bonds can also be readily observed.
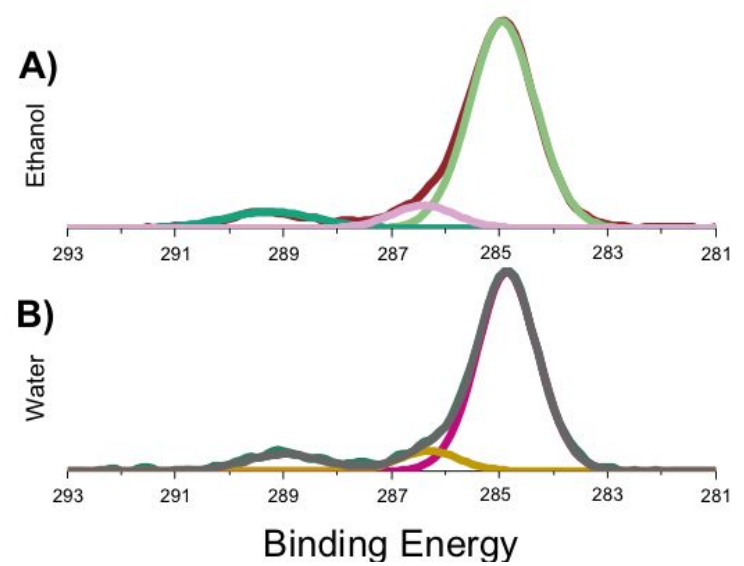

Figure S8. XPS spectra in the C1s region of eGaIn LMPs made in the presence of A) pure ethanol or B) pure water. 


\section{References:}

1. Muruganandham, M.; Amutha, R.; Wahed, M. S. M. A.; Ahmmad, B.; Kuroda, Y.; Suri, R. P. S.; Wu, J. J.; Sillanpää, M. E. T., Controlled Fabrication of $\alpha-\mathrm{GaOOH}$ and $\alpha-\mathrm{Ga} 203$ Self-Assembly and Its Superior Photocatalytic Activity. The Journal of Physical Chemistry $C$ 2012, 116 (1), 44-53.

2. $\quad$ Kumar, V. B.; Mishra, R. K.; Pulidindi, I. N.; Porat, Z. e.; Luong, J. H. T.; Gedanken, A., Preparation and Catalytic Activity of Thermosensitive Ga2O3 Nanorods. Energ Fuel 2016, 30 (9), 7419-7427.

3. Lertanantawong, B.; Riches, J. D.; O’Mullane, A. P., Room Temperature Electrochemical Synthesis of Crystalline GaOOH Nanoparticles from Expanding Liquid Metals. Langmuir 2018, 34 (26), 7604-7611.

4. $\quad$ Bartram, S.; Moffatt, W.; Roberts, B., The In-Sn phase diagram and superconductivity in In3Sn. Journal of the Less Common Metals 1978, 62, 9-12.

5. $\quad$ Bilodeau, R. A.; Zemlyanov, D. Y.; Kramer, R. K., Liquid metal switches for environmentally responsive electronics. Advanced Materials Interfaces 2017, 4 (5), 1600913.

6. $\quad$ Dickey, M. D.; Chiechi, R. C.; Larsen, R. J.; Weiss, E. A.; Weitz, D. A.; Whitesides, G. M., Eutectic gallium-indium (EGaIn): a liquid metal alloy for the formation of stable structures in microchannels at room temperature. Adv Funct Mater 2008, 18 (7), 10971104.

7. Kim, D.; Thissen, P.; Viner, G.; Lee, D.-W.; Choi, W.; Chabal, Y. J.; Lee, J.-B., Recovery of Nonwetting Characteristics by Surface Modification of Gallium-Based Liquid Metal Droplets Using Hydrochloric Acid Vapor. Acs Appl Mater Inter 2013, 5 (1), 179-185.

8. $\quad$ Epp, J. M.; Dillard, J., Effect of ion bombardment on the chemical reactivity of gallium arsenide (100). Chem Mater 1989, 1 (3), 325-330.

9. Hamza, M. H. A.; Serratrice, G.; Stebe, M. J.; Delpuech, J. J., Solute-solvent interactions in perfluorocarbon solutions of oxygen. An NMR study. J Am Chem Soc 1981, 103 (13), 3733-3738.

10. Lin, Y.; Cooper, C.; Wang, M.; Adams, J. J.; Genzer, J.; Dickey, M. D., Handwritten, soft circuit boards and antennas using liquid metal nanoparticles. Small 2015, 11 (48), 63976403. 